\title{
JHOMME LHomme
}

L'HOMME Revue française d'anthropologie

$189 \mid 2009$

Oralité et écriture

\section{"Il n'existe pas de pauvres chez nous"}

De la supériorité des nomades sur les sédentaires

Jean-François Gossiaux

\section{(2) OpenEdition \\ Journals}

Édition électronique

URL : http://journals.openedition.org/lhomme/22002

DOI : 10.4000/lhomme.22002

ISSN : 1953-8103

Éditeur

Éditions de l'EHESS

Édition imprimée

Date de publication : 1 janvier 2009

Pagination : 115-138

ISSN : 0439-4216

Référence électronique

Jean-François Gossiaux, " "Il n'existe pas de pauvres chez nous" », L'Homme [En ligne], 189 | 2009, mis en ligne le 01 janvier 2011, consulté le 19 avril 2019. URL : http://journals.openedition.org/ Ihomme/22002 ; DOI : 10.4000//homme.22002 


\title{
"Il n'existe pas de pauvres chez nous"
}

De la supériorité des nomades sur les sédentaires

\author{
Jean-François Gossiaux
}

I

ORDRE DES ÉTATS-NATIONS censé avoir régi le monde durant les deux derniers siècles est fondamentalement un ordre sédentaire, un ordre conçu par et pour des sédentaires. Certes, si l'on en croit Ernest Gellner, l'émergence du nationalisme est liée au passage de la société agraire à une société industrielle fondée sur la mobilité des individus. Mais, outre que la plupart des nations de l'industrialisme triomphant sont longtemps restées des nations de paysans, au moins d'un point de vue démographique, les techniques administratives de leurs États tout autant que leur symbolique politique ou que leur appareillage idéologique ont érigé en modèle normatif l'attachement local, y compris pour des populations ouvrières soumises aux fluctuations géographiques de la stratégie industrielle (Gossiaux 1992). Les sujets ne sont plus attachés à la glèbe, mais il n'est de bon citoyen, ou de bon ressortissant, que localisé. Le pouvoir lui-même a abandonné ses habitudes nomades ${ }^{1}$ pour s'enfoncer dans la centralité fonctionnelle des capitales.

Peut-on faire l'hypothèse que l'effacement relatif - et lui-même hypothétique - des États-nations, avec la "globalisation ", va s'accompagner de l'émergence ou de la ré-émergence de certains éléments ressortissant, sinon au nomadisme, du moins à un modèle moins marqué au sceau de la sédentarité ? Dans Le Gouvernement du monde. Une critique politique de la globalisation (2004), Jean-François Bayart souligne à cet égard le rôle

1. Les touristes qui se pressent dans les salles vides des châteaux de la Loire ne peuvent que constater de visu les effets décevants du nomadisme royal, qui a perduré en France jusqu’à la Renaissance. Le même phénomène a existé jusqu’à une période plus récente dans un pays comme le Maroc: "Le trône du roi est sa selle, le ciel son baldaquin » (proverbe cité par C. Geertz [1986 : 171]). En Europe même, Marc Abélès, dans son ethnographie du Parlement européen, observe la réapparition d'une sorte de mobilité géographique des instances du pouvoir entre Strasbourg, Luxembourg et Bruxelles (Abélès 1992). Mais il est vrai que ce pouvoir est européen et non plus national. 
remarquable sinon central des peuples «véhiculaires " situés aux frontières (sociales et géographiques). Des peuples qui se sont souvent constitués comme tels à partir de leur fonction d'intermédiation, en particulier marchande. Des peuples souvent "marginaux» qui ont une pratique sociale du déplacement et qui sont intégrés de longue date dans les marchés mondiaux. Des observations personnelles réalisées à l'occasion d'enquêtes récentes ${ }^{2}$ vont dans un sens analogue, en faisant apparaître de manière convergente la supériorité locale de populations anciennement nomades, c'est-à-dire la position dominante et centrale que celles-ci tendent à occuper par rapport à leur environnement social.

C'est cette convergence que je voudrais interroger ici, sans prétention à l'universalité et au-delà des considérations générales sur la mondialisation et le nomadisme, sur la mondialisation et les marges ${ }^{3}$. La question est celle des modalités effectives - i. e. des conditions - de cette supériorité, ou de cette centralité paradoxale, notamment dans une situation de transition telle que l'actuelle mondialisation. En l'occurrence, mon interrogation portera sur une transition particulière, celle qui affecte les sociétés anciennement socialistes ${ }^{4}$. Une transition, donc, multiple: du nomadisme à la sédentarité ; du socialisme au postsocialisme, sinon au capitalisme; le tout sur fond de transition "globale», ou prétendue telle.

Je le ferai à partir de trois cas, de trois populations ayant chacune un passé de pastoralisme nomade ou semi-nomade et une histoire récente marquée par le socialisme, mais par un socialisme à visage dans chaque cas différent. Deux de ces populations ont en commun d'avoir conduit leurs troupeaux à travers les Balkans du temps de l'empire ottoman et de s'être fixées dans les différents États-nations qui se sont partagé la péninsule : les Valaques (Aroumains) en Macédoine, Albanie, Grèce, Bulgarie, Serbie, Roumanie; les Saracatsans (ou Karakatchans) en Grèce et Bulgarie.

2. Ces enquêtes ont été notamment menées en Russie (Sibérie, Territoire d'Aga) entre 2001 et 2005 et en Bulgarie en 2004. Je ferai également référence à des faits que j’ai observés lors de recherches plus anciennes, en 1997 (Bulgarie), 1993 (République de Macédoine), 1987 (Yougoslavie, Macédoine). Les enquêtes dans le territoire d'Aga ont été effectuées dans le cadre de l'Action concertée incitative du CNRS "Terrains, techniques, théories ", projet Nomadisme, identité, ethnicité : espaces et pouvoirs locaux (Russie, Kirghizstan, Niger, Mauritanie).

3. Nomadisme et marges ne se confondent évidemment pas, même s'ils ne sont pas sans rapport: les diverses acceptions du terme slave vlah (cf. infra) montrent bien que nomadisme est parfois synonyme, au sens propre, d'étrangeté, de marginalité.

4. Sur les différentes acceptions du terme et sur les différentes approches théoriques, celles qui mettent l'accent sur les notions de système et de rupture, sans jugement de valeur, et celles qui sont portées par les notions de modernité et de progrès, cf. Sociologie et sociétés, 1990, 22 (1), en particulier l'article de Jacques Hamel \& Mohammed Sfia, "Sur la transition » et celui de Maurice Godelier, "La théorie de la transition chez Marx ». Pour une critique de la transitologie postsocialiste concevant la transition comme un passage univoque du socialisme au marché et du totalitarisme à la démocratie, cf. Burawoy \& Verdery (1999), en particulier «Introduction » : 1-19. 
À l'autre bout du continent eurasiatique, le XXe siècle a vu les Bouriates d'Aga passer de l'empire russe à l'actuelle Fédération de Russie, via les différents avatars du régime soviétique. Ces trois histoires sont autant d'illustrations archétypiques de l'«encastrement " du procès économique dans le politique ${ }^{5}$. La question devient donc: comment et selon quelle logique des populations a priori infériorisées par des institutions et des représentations politiques fondées sur la sédentarité (celles des Étatsnations aussi bien que celles de l'URSS, à quoi s'est ajoutée, dans le cas des Bouriates, une répression stalinienne particulière) se retrouvent-elles dans une situation de suprématie locale lorsqu'elles sont passées à l'état d'« exnomades" ? Le passé nomade n'est-il pas constitutif d'une qualité spécifique hors de la sédentarité banale? Si oui, la suprématie locale lui est-elle liée, et selon quelle logique ? Et, avant tout, quelle réalité a historiquement recouverte cette supposée minorisation des nomades? Ceux-ci ne trouvaient-ils pas, dans le système, des ressources propres dont certaines ont pu être conservées dans le processus de transition?

\section{Les Valaques}

Les Valaques ne sont pas (ou pas seulement; en tout cas, ce n'est pas l'acception que nous retiendrons ici) les habitants de la Valachie, principauté dont l'union avec la Moldavie a formé la Roumanie. En première définition, nous dirons qu'il s'agit d'une population archéo-balkanique romanisée. Étymologiquement, le terme serait d'origine germanique, avec des correspondances actuelles en allemand (Welsch: latin, roman, étranger), en anglais (Wales: Gallois), en français (Wallon), en polonais (Wolosy: Roumain)... Il aurait désigné initialement une tribu celte voisine des Germains, puis tous les Celtes, puis les Gallo-romains, puis tous les peuples romans. Dans sa signification ethnique actuelle (sous sa forme slave: Vlah, pluriel Vlasi), il s'applique de manière générique à l'ensemble des populations de langue romane disséminées au sud du Danube.

Valaque constitue un exemple, parmi d'autres, de la contamination réciproque des désignations ethniques et professionnelles : les vlasi, ce sont les bergers (nomades). Cette correspondance sémantique est générale dans les Balkans. Ainsi, à côté de Vllah, une forme albanaise de l'ethnonyme est Coban, mot turc qui signifie "berger». Une autre acception professionnelle de vlah est "caravanier». Par ailleurs, la signification ethnique s'étend de manière générique à l'autre, à l'étranger (sur le même mode que la forme allemande Welsch), avec fréquemment une connotation péjorative.

5. Je reprends ici la notion d'embeddedness développée par Karl Polanyi (Polanyi \& Arensberg 1975). 
Les "Valaques", ce sont les Serbes pour les Croates, les gens de la montagne pour ceux de la côte, etc.

II8 De façon cohérente avec ce qui précède, et selon un phénomène assez banal, Valaque est un ethnonyme attribué de l'extérieur, un exonyme. Les endonymes sont, selon les cas, "Aroumains" (Armeni) ou "Roumains" (Rumeni). Les Valaques « roumains" sont les Valaques proches du Danube (en Bulgarie et Serbie). Les Aroumains sont quant à eux présents dans l'ensemble de la péninsule, surtout en Macédoine, en Grèce et en Albanie. Ce sont eux qui sont descendants de pasteurs nomades (ou seminomades), ou en tout cas se revendiquent comme tels, et c'est à eux que nous nous intéresserons ici.

Les Aroumains pratiquaient traditionnellement une transhumance inversée, l'habitat "fixe ", dans des cabanes (tenda), étant celui d'été, dans la montagne, alors que les installations d'hiver variaient au hasard des occupations de maisons abandonnées, dans la plaine, sur les côtes de la Grèce et jusqu'aux rivages de la mer Noire ${ }^{6}$. Fournissant en laine l'armée turque, ils jouissaient de privilèges de circulation au sein de l'empire ottoman et, en échange de taxes acquittées sur les têtes de bétail, ils pouvaient disposer des meilleurs pâturages, entre 1000 et 1500 mètres. (Les Bulgares ${ }^{7}$, moins riches, devaient se contenter de faire pâturer leurs bêtes plus bas, plus près des villes, et ne transhumaient pas.) L'élevage des moutons s'accompagnait de celui des chevaux, comme animaux de bât ou de trait et à l'occasion pour la vente. La transhumance pouvait réunir plusieurs communautés familiales (agnatiques), sous la direction d'un kikaja chargé des rapports avec l'extérieur (administratifs, commerciaux, etc.) ${ }^{8}$.

Les guerres balkaniques, en érigeant de nouvelles frontières étatiques, entre la Grèce et la Yougoslavie notamment, perturbèrent voire coupèrent les parcours habituels des pasteurs. Ainsi débuta un processus de sédentarisation, en particulier dans la Macédoine yougoslave. Les Aroumains s'y fixèrent sur leur habitat d'hiver et commencèrent à y cultiver la terre, passant ainsi à un agro-pastoralisme semi-nomade. L'érection de la Fédération socialiste yougoslave au lendemain de la Seconde Guerre mondiale marqua une nouvelle rupture. Dans le discours commun des années 1990,

6. Les informations sur la tradition pastorale des Valaques données ici sont tirées de la littérature ethnographique (voir en particulier Antonijevic [1982] et les Cahiers du Centre d'étude des civilisations de l'Europe centrale et du Sud-Est, 1989), corroborée par des récits recueillis au cours d'enquêtes personnelles, notamment en 1997 dans la région de Velingrad (Bulgarie).

7. Bulgare est en l'occurrence un ethnonyme désignant sous les Ottomans l'ensemble des slavophones orthodoxes de la Macédoine (Macédoine de l'Égée, actuellement partie de la Grèce, Macédoine du Vardar, actuelle République de Macédoine, Macédoine du Pirin, actuellement partie de la Bulgarie). 8. Il ne s'agit pas d'organisations tribales telles qu'il en existait chez les Monténégrins ou les Albanais. 
après l'implosion de la Yougoslavie et du socialisme, la collectivisation est l'apocalypse dans laquelle s'est anéanti le monde valaque. Les troupeaux furent confisqués par les coopératives. Les pasteurs aroumains, qui ne voulaient pas rester dans les propriétés collectives, abandonnèrent l'élevage. Rien ne subsista de la vie passée.

La réalité, cependant, ne fut pas aussi uniforme qu'elle apparaît dans le récit postcommuniste. C'est du moins ce que laisse à penser le cas contreexemplaire du village de $S$., au cour de l'actuelle République de Macédoine (Gossiaux 2002 : 150-157). Dans ce village valaque fut fondée en 1946, sur le mode kolkhozien, une "coopérative rurale de travail" (seljacka radna zadruga), à l'initiative d'un " activiste " qui occupait d'importantes fonctions politiques au niveau régional, et qui se trouvait par ailleurs être le fils du chef traditionnel de la communauté villageoise ${ }^{9}$. Tous les villageois remirent leurs bêtes et leurs terres à la coopérative. L'argument utilisé pour les convaincre était sémantique : il s'agissait simplement de passer d'une zadruga familiale à une zadruga socialiste ${ }^{10}$, sans que cela tire autrement à conséquence. L'opération s'accompagna de l'arrivée au village de plusieurs familles, en majorité macédoniennes ${ }^{11}$, qui par un artifice de procédure devinrent membres de la coopérative. La zadruga de S. devint l'un des fleurons les plus visités du socialisme de Macédoine. Elle survécut à la décollectivisation de 1953 et ne disparut qu'en 1980, au grand dam des villageois.

Les pasteurs valaques qui ne s'intégrèrent pas ainsi dans l'ordre socialiste des campagnes ne furent pas pour autant marginalisés. Utilisant des possibilités financières auxquelles avaient grandement contribué leur traditionnelle méfiance à l'égard du papier-monnaie et leur habitude de thésauriser en or, nombre d'entre eux achetèrent des commerces en ville, firent faire des études à leurs enfants et ne furent pas les moins biens lotis de l'ordre économique yougoslave.

Quelle qu'en soit la cause, proprement politique, avec la collectivisation, ou relevant de l'évolution économique globale, la disparition du pastoralisme semi-nomade réduit désormais le terme valaque à sa dimension ethnonymique. Il existe bien aujourd'hui encore, en Macédoine, un élevage ovin plus ou moins marginal auquel les Valaques ont toujours leur part, mais les modalités en ont profondément changé. Les éleveurs résident généralement en ville, et ils se mettent à plusieurs pour salarier un berger - souvent un Turc. Pour résiduelle qu'elle soit, cette activité

9. La communauté villageoise se confondait, en l'occurrence, avec une communauté familiale.

10. Zadruga signifie à la fois communauté familiale et coopérative. Il s'agit d'un terme d'origine savante, apparu dans sa première acception au XIX siècle dans le dictionnaire de Vuk Karadzic.

11. Macédoniens est pris ici au sens ethnico-national. La constitution de la République de Macédoine distingue les «Macédoniens » et « les autres nationalités qui y habitent ». 
contribue à entretenir certaines représentations archétypiques. Ainsi, dans les années 1990, le plus grand marchand de moutons de Macédoine était un Aroumain de Veles. En lui étaient réunies les deux images traditionnelles du Valaque : celle du pasteur et celle du citadin fortuné.

Il existe un lien logique entre ces deux images : celui qui relie l'élevage et le commerce. L'un et l'autre participent en l'occurrence d'une même chaîne économique articulée sur un même mode de vie itinérant. Rappelons que "valaque ", en même temps que "pasteur nomade", signifie "caravanier". Si les Aroumains pratiquaient assez naturellement le commerce des produits directement dérivés de l'élevage (tissus, peaux), leurs caravanes pouvaient tout aussi bien transporter soie, tabac, café, huile... ${ }^{12}$. Le trafic connut un essor important à partir du XVII ${ }^{\mathrm{e}}$ siècle et contribua au développement et à la prospérité des bourgades et des villes, essentiellement aroumaines, du nord de la Grèce, de la Macédoine et de l'Albanie. Détenant le quasimonopole du tissage et du commerce de la laine, contrôlant la plus grande partie du négoce entre la Turquie et l'Autriche, les Valaques ouvrirent des comptoirs à Vienne, Leipzig, Novi Sad, Venise... Les débuts du capitalisme en Autriche renforcèrent encore la tendance à l'émigration - une émigration élitaire : gros négociants, banquiers, médecins... - à l'origine de véritables dynasties européennes, comme les Sina ou les Averoff, rendus célèbres au XIX siècle par leur soutien à la cause hellénique.

Un événement rompant le fil pacifique de l'histoire va être, nonobstant l'ancienneté de cette tradition migratoire, mythifié en catastrophe fondatrice de la diaspora aroumaine : le sac, en 1788, de Moschopole ${ }^{13}$, la plus grande ville valaque de l'époque, dans le contexte de la guerre russo-turque et de l'anarchie affectant alors la région. Les familles les plus riches allèrent s'installer dans les grandes villes européennes, dans le courant du mouvement déjà engagé. Les familles ordinaires reproduisirent celui-ci à l'échelle des Balkans. Beaucoup se fixèrent dans l'actuelle République de Macédoine, notamment dans la ville de Krusevo. Les autres essaimèrent dans l'ensemble de la péninsule, en particulier en Serbie, où ils furent appelés "Tsintars» (Cincari). Sous le régime ottoman, ceux-ci y étaient pratiquement les seuls citadins non turcs, avec les Slaves islamisés (d'ailleurs appelés eux-mêmes "Turcs») et les juifs. Une thèse soutenue en Serbie même en fait le noyau de la bourgeoisie serbe ${ }^{14}$.

12. Cf. M. Cazacu, «Les Valaques dans les Balkans occidentaux (Serbie, Croatie, Albanie, etc.). La pax ottomanica (XVe-XVII siècles)", Cahiers du Centre d'étude des civilisations de l'Europe centrale et du Sud-Est, 1989, 9 : 79-93.

13. Aujourd'hui le village de Voskopoje, en Albanie.

14. Dusan Popovic, O Cincarima. Prilozi pitanju postanka nase carsije (= Des Tsintsars. Contribution à la question de l'origine de notre bourgeoisie), Belgrade, 1937. 
D'une façon générale, et si l'on excepte certaines implantations relativement massives comme celle de Krusevo, les installations se faisaient de façon dispersée, par petits groupes et en séparant assez systématiquement les parentèles. Cette pratique - sinon cette stratégie - évitait la concurrence interne et était propice à la formation de réseaux. On trouvait des Valaques dans tous les métiers du commerce et de l'artisanat (notamment dans la construction), avec, en particulier, un quasi-monopole de l'hôtellerie.

Qu'en est-il aujourd'hui de la présence (et de la visibilité) valaque dans les Balkans, étant entendu, comme nous l'avons indiqué plus haut, que l'acception pastorale qui était l'autre versant de l'ethnonyme est pratiquement renvoyée dans le passé ? Cette présence est explicite et même officielle en République de Macédoine, puisque les Valaques sont mentionnés dans le préambule de la constitution comme l'un des peuples «qui y habitent ». Krusevo est considérée comme la ville valaque de Macédoine. La moitié de la population y est valaque, dit-on à Skopje. Et, effectivement, la quasi-totalité des personnes qu'on y rencontre sont, soit valaques, soit "à moitié» valaques, soit mariées à un(e) Valaque. Les pratiques linguistiques apparaissent étroitement liées aux relations inter- et intragénérationnelles. Le macédonien est la langue utilisée avec les enfants, et le valaque est la langue exclusive des relations avec la génération âgée. Cependant, tous les vieux parlent le grec, qui était la langue véhiculaire des Aroumains - beaucoup ont été alphabétisés en grec et ont suivi l'école grecque ${ }^{15}$.

Si la moitié de la population de Krusevo est censée être valaque, seuls $15 \%$ sont recensés comme tels ${ }^{16}$. Le même phénomène se retrouve au niveau de la république. La population valaque y est estimée (par les Valaques eux-mêmes) à environ 150000 individus - 120000 au minimum. Or son effectif tel qu'il ressort des statistiques officielles n'est que de $7764^{17}$. Comment interpréter une telle sous-estimation, qui est en fait une autominoration, puisque les données du recensement sont déclaratives?

Une explication indigène, avancée notamment dans les familles mixtes, est qu'il est plus commode de se déclarer macédonien. En fait d'explication, il s'agit plutôt d'une façon de déplacer la question : en quoi l'identité valaque est-elle malcommode, et pourquoi est-elle dominée par l'identité concurrente? Une autre raison mise en avant est qu'il était mal vu de se

15. Une école roumaine a été créée au début du siècle, mais elle ne s'est pas pérennisée.

16. Recensement de 1991.

17. Recensement de 1991. Les recensements précédents donnent 22000 en 1971 et 32000 en 1981. Les recensements ultérieurs (au déroulement contesté, notamment, par la minorité albanaise) donnent 8200 en 1994 et 8100 en 2002. 
déclarer valaque sous le régime communiste. En l'occurrence, c'est la véracité de l'affirmation qui apparait incertaine. Comme nous l'avons vu, il a pu exister de belles carrières communistes, au moins au niveau local, pour des Valaques connus comme tels. Et le fait que le régime lui-même proposait la catégorie "valaque» au recensement n'atteste pas une hostilité patente. Il est d'ailleurs à noter que l'insuccès de cette catégorie s'est notablement accentué après la fin du communisme. Selon un troisième type d'explication, enfin, la déclaration valaque serait socialement peu valorisante. L'image des Valaques est celle de nomades à la limite de la sauvagerie, celle de femmes vêtues de noir et au front tatoué d'une croix ${ }^{18}$. Mais c'est aussi, à l'inverse, celle du bourgeois fortuné, du commerçant avisé. Une image effectivement peu adaptée a priori au socialisme, mais certainement moins incongrue dans l'après-socialisme.

En fait, au-delà de ces rationalisations indigènes et contextuelles, la sous-déclaration systématique aux recensements renvoie à un phénomène plus général. L'identité valaque a toujours été mise en veilleuse et, a fortiori, n'a jamais été objet de revendication. L'entité valaque n'a jamais constitué un sujet politique. En témoigne l'insuccès du mouvement aroumain, soutenu par la Roumanie, dans le champ clos des affrontements nationalitaires qu'était la Macédoine du début du XXe siècle. La tentative d'instaurer une Église autonome, à l'instar des autres Églises nationales, fit long feu, de même que végéta, pour finalement disparaître, le réseau d'enseignement aroumano-roumain ${ }^{19}$. Il n'est meilleur Grec qu'un Valaque grec, meilleur Serbe qu'un Valaque serbe.

Cela, évidemment, est à mettre en rapport avec les «niches économiques » occupées historiquement par les Aroumains, pasteurs nomades ou commerçants au long cours, en tout cas constamment en situation de contact. Cette situation a naturellement engendré une polyglossie qui peut expliquer la faible attractivité des écoles en langue vernaculaire. Elle a aussi développé une capacité d'adaptation au milieu, passant notamment par une adaptation au pouvoir. «Lors du massacre de Batak [un épisode du soulèvement bulgare de 1876] les femmes marquées d'une croix furent épargnées par les Turcs, au motif que les Valaques ne font pas de politique. » L'anecdote

18. Les femmes valaques portent traditionnellement, dans certaines régions, des croix dessinées sur les poignets ou sur le front. Sur l'ambiguïté des Aroumains vis-à-vis du pastoralisme nomade, cf. Gossiaux (2002: 157-170).

19. L'enseignement se faisait en aroumain dans les petites classes, ensuite en roumain littéraire (daco-roumain). Sur le mouvement aroumain au tournant des XIXe et XXe siècles, voir M. D. Peyfuss "Les Aroumains à l'ère des nationalismes balkaniques ", Cahiers du Centre d'étude des civilisations de l'Europe centrale et du Sud-Est, 1989, 9: 129-149. 
m’a été rapportée en 1997 par un Aroumain de Bulgarie. Elle lui paraissait révélatrice d'une attitude générale dont il n'y avait à tirer ni honte ni fierté, mais qui, l'exemple le montrait, pouvait présenter quelques avantages.

Cependant, depuis quelques années, les Valaques se signalent par un foisonnement de manifestations identitaires à tous les niveaux, laissant à penser que cette population qui faisait jusqu'alors de la discrétion une règle de conduite a opéré un renversement stratégique. Le phénomène concerne essentiellement la République de Macédoine, pays où leur place est le plus officiellement consacrée. Nous avons vu que la constitution les mentionnait explicitement dans son préambule. Celui-ci fait également référence à la République de Krusevo ${ }^{20}$, conférant ainsi une valeur emblématique à leur ville. La radio et la télévision diffusent régulièrement des émissions en valaque. Ces émissions sont sous-titrées en macédonien, et le jeu des ethnonymes endogènes et exogènes trouve ici une remarquable illustration : "aroumain" (armen) - y est traduit par "valaque» (vlah). Plusieurs villes comptent des associations valaques, fédérées en une «ligue» au niveau de la république.

La sphère privée et la sociabilité informelle se trouvent de la même façon investies par cette identité renouvelée. Des ascendances valaques insoupçonnées se révèlent. Des conversations se tiennent ostensiblement en valaque. Tout ce qui compte à Skopje, et au-delà, se voit attribuer (ou s'attribue) des origines valaques : banquiers, hommes d'affaires, propriétaires d'agences de voyages (sans parler des hommes politiques ni des intellectuels)... Dans ce palmarès de la notabilité, on retrouve en fait, sous une forme moderne, les professions traditionnelles des Aroumains. Les stéréotypes négatifs dont ils étaient (ou se croyaient) gratifiés se transmuent en valeurs positives. L'appât du gain devient esprit d'entreprise, la richesse signifie la compétence, l'arrivisme s'appelle dynamisme. La stigmatisation de classe censée avoir prévalu durant la période socialiste est inversée. La bourgeoisie est à la mode, et, par voie de conséquence, l'identité valaque l'est aussi.

Le problème, cependant, est de lui trouver des marqueurs ou, pour utiliser la terminologie de Fredrick Barth, de relever des frontières ethniques délabrées. Le pastoralisme, qui constituait l'essence de la valaquité, ne vaut plus que par référence au passé et n'a plus présentement de pertinence. La fraction urbaine des Aroumains s'est quant à elle toujours fondue dans l'environnement, s'habillant et vivant «à la franque» ou à la turque, et donc

20. Éphémère embryon d'État érigé en 1903 par les insurgés de la saint Élie (Ilinden) et qui avait son siège dans la ville éponyme. 
n'est d'aucune ressource en matière de spécificité visible. Quant à la langue, sa valeur emblématique souffre des défauts habituels dans ce genre de situation : perte de compétence des jeunes générations, hétérogénéité dialectale et rivalité entre régions pour l'imposition des normes d'écriture ${ }^{21}$.

En dépit de ces difficultés, ou à cause d'elles, l'affirmation identitaire se joue sur un mode volontariste, voire activiste, jusqu'à atteindre une dimension proprement politique. (Le programme de la ligue valaque comporte une référence à la "réalisation des droits de l'homme", litote habituelle pour signifier cette dimension. ${ }^{22}$ Il s'agit donc d'une affirmation de type ethniciste - si l'on définit l'ethnicisme comme la valorisation politique de l'ethnicité. Mais, en l'occurrence, cet ethnicisme n'a rien à voir avec un quelconque ethno-nationalisme. Il relève d'une relation spécifique au territoire et à l'État. La revendication aroumaine ne vise pas à un État-nation ni même à une autonomie territoriale. Elle ne participe pas non plus d'une attitude de "minorité nationale", à la différence, par exemple, des Albanais, qui négocient leur participation à l'État macédonien tout en gardant un œil sur leurs compatriotes de l'autre côté des frontières de la république. Les Aroumains ne se veulent pas les enfants d'une lointaine mère-patrie roumaine.

Il existe, dans l'affirmation valaque telle qu'elle se manifeste notamment en Macédoine, deux dimensions qui, bien qu'apparemment contradictoires, expriment l'une et l'autre le fait que la qualité «valaque» ne prend sens que dans un environnement, qu'elle n'a pas de valeur en soi (il n'y a pas d'identité valaque intrinsèque) mais signifie seulement la possibilité d'une relation (il n'y a qu'un rapport "valaque " au monde).

La première dimension est celle du nationalisme macédonien. Les Aroumains se considèrent comme des Macédoniens, selon une conception territoriale de l'appartenance nationale («Nous sommes d'ici») qui va au rebours de la définition ethnique de la république donnée par la Constitution ( "l'État national du peuple macédonien », où " habitent » d'autres nationalités, dont les Valaques). Cela explique d'ailleurs leur malaise - et leur sous-déclaration - face à des recensements précisément censés étayer cette définition ethnique. Ils se considèrent même comme les meilleurs des Macédoniens, l'élite de la Macédoine. Ils descendraient d'une population très ancienne, aussi voire plus autochtone que les Grecs, et qui aurait

21. En l'occurrence, la rivalité oppose les parlers de Stip et de Krusevo. Le valaque parlé à la télévision et à la radio est une sorte de pidgin qui ne peut satisfaire personne.

22. Si la ligue qui regroupe les associations au niveau de la Macédoine s'attribue implicitement une vocation politique, un parti politique stricto sensu peine à émerger de manière significative, à la différence de ce qu'il en est pour d'autres groupes ethniques comme les Albanais, bien sûr, mais aussi les Turcs, les Roms, voire les Serbes. 
quelque chose à voir avec la Macédoine antique. (On aurait retrouvé sur le tombeau de Philippe II des mots valaques.) Ils sont ainsi les garants d'une identité macédonienne spécifique, à l'encontre des contestations bulgare (pour la Bulgarie, les Macédoniens sont des «Bulgares ethniques») et grecque (pour la Grèce, la République de Macédoine usurpe le nom d'une région grecque). Mais leur statut d'élite, ils l'ont également gagné dans la lutte de libération nationale, notamment pendant l'insurrection d'Ilinden (1903) et la République de Krusevo. Il n'est meilleur Macédonien qu'un Valaque macédonien.

L'ethnicisme aroumain, cependant, confere à l'affirmation valaque une seconde dimension, à la fois idéologique et organisationnelle, une dimension explicitement transnationale. La ligue qui fédère les associations macédoniennes est une composante d'une ligue balkanique, qui s'inscrit ellemême dans une ligue mondiale. En fait, la plupart des manifestations «balkaniques» ou "mondiales» (principalement des congrès ou autres rencontres " culturelles ») sont organisées depuis la Macédoine, ou s’y déroulent. À côté de leur objet officiel, ces activités remplissent une fonction réticulaire aussi implicite qu'évidente. Comme la parenté, l'ethnicité représente une ressource privilégiée pour la constitution de réseaux qui sont autant de supports potentiels pour le commerce et les affaires. L'exploitation de cette ressource n'est pas chose nouvelle pour les Valaques, dont nous avons vu que les installations dans les villes balkaniques et européennes se faisaient systématiquement sur le mode de l'essaimage et de la dispersion. La nouveauté est la nécessité de sauvegarder cette ethnicité dont les marqueurs culturels tendent désormais à s'effacer. Cela passe par une institutionnalisation de la "culture» valaque et par sa protection politique, selon un schéma en tous points similaire à celui décrit par Ernest Gellner à propos des États-nations : érection d'une culture lettrée à partir d'éléments de cultures vernaculaires et imposition d'un toit politique sur cette "haute culture». Simplement, ce toit ne sera pas un État matérialisé sur un territoire, mais une entité idéelle déployée dans un espace transnational.

Le premier combat de l'ethnicisme aroumain est donc linguistique. "On essaie de garder la langue pour garder les Aroumains ", me déclare un militant d'une association de Bulgarie ${ }^{23}$. Il faut sauver ce qui subsiste de parlers en déshérence, avant d'élaborer, à coups de grammaires et de traités normatifs, la langue écrite qui donnera à la haute culture valaque ses lettres de noblesse. Le travail savant, cependant, ne vaut qu'étayé par le volontarisme politique : dans le programme de la ligue (cf. supra), "protection des cultures » et «réalisation des droits de l'homme " apparaissent comme les 
deux faces de la même médaille. S’engage alors un processus dont le caractère générique dépasse largement le cas valaque. Les associations et autres ONG spécialisées recherchent l'appui des institutions internationales, en particulier du Conseil de l'Europe, pour édifier l'abri politique qui garantira la pérennité de leur entreprise. Dans la terminologie du nouvel ordre européen, cet abri s'appelle "minorité ». En l'occurrence, l'action des ligues et associations valaques n'a pas été sans résultat. En 1997, l'Assemblée parlementaire du Conseil de l'Europe a adopté la Recommandation 1333 relative à la langue et à la culture aroumaines. Celles-ci se voient reconnue une existence de plus de deux millénaires. Un âge aussi vénérable justifie une protection, dont l'un des moyens, selon le texte, sera le statut de «minorité ethnique dispersée».

\section{Les Karakatchans}

Les Valaques ne sont pas les seuls nomades (ou ex-nomades) des Balkans. Leurs rivaux en la matière se nomment Saracatsans, une population hellénophone présente surtout en Grèce et en Bulgarie (où ils sont appelés Karakatchans) ${ }^{24}$. Cette rivalité prend parfois la forme de virulentes polémiques portant notamment sur les questions d'ethnogénèse et d'ancestralité ${ }^{25}$. Les Saracatsans disent être les plus anciens des Grecs, mais selon les Valaques de Grèce ils seraient des Valaques déromanisés. Les Valaques de Grèce se présentent quant à eux comme des Grecs romanisés, alors que ceux de Macédoine seraient des Thraco-Illyriens (également romanisés). Les Valaques de Bulgarie, comme ceux de Macédoine, affirment être des Thraces romanisés, alors que les Karakatchans seraient des Thraces hellénisés. (Cela correspond d'ailleurs à la thèse officielle en Bulgarie.) Mais les Karakatchans eux-mêmes refusent cette théorie et s'affirment Grecs.

Dans cette querelle de préséance et d'autochtonie chacun fait donc flèche de tout bois pour minorer le rival en se l'annexant. Les deux grands peuples antiques de la région, les Grecs et les Thraces, sont évidemment convoqués dans l'argumentation, mais on notera que ce sont les ancêtres «nationaux » qui constituent la référence. Les Saracatsans de Grèce se disent Grecs, les Valaques de Grèce se disent Grecs ${ }^{26}$, les Valaques de Bulgarie se disent

24. Par une confusion ethnonymique liée à l'acception professionnelle du terme vlah, les citadins qualifient parfois de "Valaques » les Saracatsans.

25. On trouve des échos particulièrement sonores de ces polémiques dans la presse grecque de la fin des années 1990.

26. Cette adhésion aux origines mythiques de l'État-nation est à mettre en relation avec le peu d'engagement des Valaques de Grèce dans les organisations valaques internationales. 
Thraces. L'exception est celle des Karakatchans de Bulgarie, qui préferent les aïeux de l'État voisin : quelle que soit sa sincérité identitaire, ce singulier souci de distinction n'est pas, nous allons le voir, dénué de bénéfices.

Les Karakatchans seraient entre 5000 et 10000 en Bulgarie. L'inexistence d'une catégorie karakatchan aux recensements entretient le flou des estimations (si tant est que les recensements puissent avoir quelque véracité statistique en la matière, ce que ne démontre pas l'exemple des Valaques précédemment évoqué). En tout état de cause, l'identification est difficile, en raison, d'une part, des mariages mixtes, désormais nombreux, et, d'autre part, d'un effet de mode qui n'est pas sans rappeler le phénomène valaque en Macédoine. Aujourd'hui, "tout le monde se découvre karakatchan ", m'affirme le maire de Kotel ${ }^{27}$. Sur les 7000 habitants de cette localité de l'est de la Bulgarie, les Karakatchans seraient environ 400. Mais ce sont eux qui tiennent le haut du pavé, au point que Kotel est, de notoriété générale, une ville karakatchan. Ils contrôlent le pouvoir politique, notamment la mairie, et possèdent les hôtels, les cafés et la plupart des entreprises locales (dont quelques-unes, dans le domaine alimentaire, ont une certaine envergure). L'élevage de moutons et les activités associées, comme la production fromagère, conservent (ou retrouvent) une existence non marginale. Ainsi, l'homme d'affaires le plus puissant de la ville est également le propriétaire d'un important troupeau.

Les Karakatchans sont restés des pasteurs transhumants jusqu'en 1958, date de la collectivisation et de la sédentarisation. Ils vivaient dans des cabanes (dans des tentes durant la dizaine de jours que durait la transhumance), l'été dans les montagnes, l'hiver dans les plaines, notamment du côté de la mer Noire. Avant 1929, les frontières étaient ouvertes et la migration d'hiver pouvait se poursuivre jusqu'en Turquie. L'organisation pastorale était semblable à celle des Valaques. La transhumance s'effectuait par groupes d'une dizaine d'unités familiales en moyenne, sous la direction d'un chef également appelé kikaja.

L'instruction et la richesse étaient hautement valorisées ${ }^{28}$ et constituaient une composante de l'identité et même de l'essence karakatchan: "Chez les Karakatchans, on ne pouvait pas se marier si on n'était pas lettré. » Pendant qu'ils gardaient les troupeaux, les aînés apprenaient l'alphabet à leurs cadets, en gravant au canif les lettres dans du bois. L'image

27. Enquête réalisée en 2004, avec l'aide d'Ivaylo Ditchev. Les informations données ici sur le pastoralisme karakatchan proviennent essentiellement d'entretiens que j'ai effectués à Kotel à cette occasion.

28. La valorisation de l'instruction reste tout aussi actuelle que celle de la richesse. Selon un informateur (karakatchan), il existe une étude montrant que les Karakatchans sont le troisième groupe quant au niveau d'instruction, après les juifs et les Arméniens. 
bucolique renvoie cependant à une réalité plus sévère : avant le mariage, les proches de la fiancée faisaient passer un test de lecture au prétendant (en grec jusque dans les années 1930, en bulgare ensuite) ${ }^{29}$. La richesse, quant à elle, fait partie encore et toujours de l'être karakatchan. "Il n'existe pas de pauvres chez nous. "Si quelqu'un est pauvre, il sort de la communauté. (Autrement dit, il se marie chez les Bulgares.)

Mais qu'en est-il des limites de la communauté (i. e. des frontières ethniques) dès lors qu'a disparu le mode de vie pastoral qui en était le principal marqueur, jusque dans l'image de la culture savante? Placés devant un problème similaire, les Valaques aroumains, nous l'avons vu, cherchent la solution dans la spécificité de leur langue. Les Karakatchans ne possèdent pas (ne peuvent ou ne veulent pas posséder) une telle ressource. Ils ne revendiquent pas une identité linguistique particulière, considérant leur parler vernaculaire comme un simple patois grec. En l'absence de matériau identitaire immédiatement disponible, donc, les frontières du groupe vont être tracées de façon administrative et bureaucratique. Concrètement, il existe une association karakatchan nationale, dotée de branches locales. Tous les Karakatchans sont censés y adhérer. Ce sont les dirigeants des sections locales qui dressent la liste des Karakatchans de leur ville (liste qui se confond de fait avec celle des membres de l'association). L'adhésion d'intrus est pratiquement impossible, dans la mesure où tout se passe dans une société d'interconnaissances. Le pouvoir de contrôle assuré par les dirigeants locaux tend naturellement à prendre une dimension morale et politique : il ne peut exister que de «bons Karakatchans». Une pression s'exercerait ainsi, selon certains, à l'encontre des mariages mixtes ${ }^{30}$.

L'objet de l'association est officiellement ethnographique et culturel. À la différence de ses homologues valaques, elle ne s'intègre pas dans un ensemble supranational, entretenant seulement des relations plus ou moins formalisées avec les associations saracatsans grecques. Une raison peut en être simplement démographique. Les Karakatchans-Saracatsans sont nettement moins nombreux que les Valaques, dans les Balkans et à l'étranger. Par ailleurs, et surtout, l'identité grecque elle-même est traditionnellement "porteuse » et propice aux fonctionnements réticulaires transnationaux. Ériger en organisation internationalement structurée une spécificité saracatsan n'a donc pas de pertinence de ce point de vue. L'abandon du parler vernaculaire à son statut de patois grec s'inscrit de

29. K.D., né en 1925, ancien pasteur nomade. Kotel, septembre 2004.

30. Cette opinion est émise par des non-Karakatchans. En fait, le membre bulgare d'un couple mixte peut être "karakatchanisé " en étant inscrit sous le nom de famille karakatchan de son conjoint et profiter ainsi des avantages de cette appartenance. (Le système anthroponymique traditionnel a été maintenu et double le système officiel.) 
façon cohérente dans ce constat. La ressource identitaire ne vaut que dans le contexte bulgare, et l'ouverture internationale qu' elle permet est en fait simplement une ouverture sur la Grèce voisine. Mais les possibilités offertes par celle-ci apparaissent considérables, le rôle de l'association en devenant du coup fondamental, bien au-delà de son prétexte culturel. L'État grec, en effet, accorde aux Karakatchans de Bulgarie un statut privilégié leur permettant d'obtenir visas, contrats de travail, bourses d'étudiants... Ce statut leur est attribué sur la base des listes que l'association fournit au consulat grec.

Le dispositif est largement utilisé par les intéressés, principalement pour des migrations temporaires. Ils se font embaucher en Grèce, souvent de façon collective, pour tous types de travaux, en particulier pour les travaux agricoles. Les notables eux-mêmes n'hésitent pas, pour l'occasion, à se faire ouvriers. L'un d'entre eux, qui a vécu cette expérience, rapporte avec complaisance l'étonnement des Grecs constatant qu'une brigade de travailleurs agricoles était pleine de diplômés, avec notamment "un vrai docteur $»^{31}$. Il s'agit là d'une manifestation, parmi d'autres, de l'aptitude des Karakatchans à faire feu de tout bois et à saisir toutes les opportunités économiques.

Cette aptitude se manifeste notamment durant les périodes de transition. Les Karakatchans en ont connues plusieurs au cours des dernières décennies. La première, consécutive à la collectivisation de 1958, fait l'objet de récits douloureux de la part de ceux qui l'ont vécue ${ }^{32}$. À la différence du cas des Valaques macédoniens évoqué plus haut, la mesure semble avoir été mise en œuvre d'une façon particulièrement brutale et traumatisante. L'événement est rapporté comme un arrachement, comme l'anéantissement d'un mode de vie spécifique et affectivement chargé. (Un informateur m'a affirmé qu'il pouvait reconnaître individuellement chacun de ses 300 moutons.) La collectivisation a marqué la fin du nomadisme qui avait perduré jusqu'alors. Le processus fut chaotique, donnant lieu à des épisodes romanesques de clandestinité et de fuite avec les troupeaux à travers le pays. Au bout du compte, cependant, les pasteurs dépossédés finirent par percevoir quelques compensations non négligeables, sous forme d'indemnités ou en réussissant à vendre plus ou moins légalement un certain nombre de bêtes. Ils entrèrent généralement comme bergers salariés dans les nouvelles organisations collectives - en subissant parfois le traumatisme supplémentaire d'être placés sous commandement féminin... La seconde grande transition est bien sûr liée à la liquidation du socialisme.

31. Kotel, septembre 2004.

32. Kotel, septembre 2004. 
Elle a en fait débuté dans les années 1980, alors que la décomposition du régime était déjà amorcée. Cette période vit les Karakatchans - ou du moins certains d'entre eux - s'enrichir considérablement, d'une manière que l'on ne peut dire inavouable, car elle est rapportée sans gêne et même avec une certaine ostentation, mais qui relève d'un rapport quelque peu distancié à la légalité. Dans un contexte de désorganisation globale, ils se dispersèrent alors à travers le pays, d'une part pour accomplir des tâches pour lesquelles ils avaient une compétence particulière (tonte, traite, etc.), d'autre part, et surtout, pour se livrer à un trafic généralisé des ovins. Celui-ci se nourrissait de la rigidité et des ratés d'une planification entraînant excédents ici et manque ailleurs, sans parler des détournements. Il bénéficia aussi, dans les dernières années de la décennie, de la résurgence des kurban, ces fêtes orthodoxes au cours desquelles sont sacrifiés des moutons dont il est religieusement malséant de marchander le prix. Les Karakatchans ont pu ainsi accumuler un capital qui n'est pas étranger à leur domination économique locale, une domination confortée, comme nous l'avons vu, par l'exploitation intensive de la « rente ethnique».

\section{Les Bouriates d'Aga}

À la double transition du nomadisme à la sédentarité et du socialisme au «capitalisme» s'est, pour les Bouriates de Sibérie méridionale, ajouté un troisième changement également fondamental puisque, avec la disparition de l'URSS, ils ont changé de pays, d'État et de citoyenneté. Cependant, quels qu'aient pu être les effets affectifs et symboliques de la perte de l'identité soviétique, la mutation fut plus théorique que réelle. Le district (okrug) autonome bouriate d'Aga est actuellement "sujet $»^{33}$ de la Fédération de Russie. Lointain successeur de la "douma de la steppe d'Aga » fondée en 1837 dans le cadre de l'empire russe, il était déjà officiellement autonome depuis 1937 dans le cadre de l'Union soviétique, au sein de la Russie. Avec ses 19000 kilomètres carrés (pour 79000 habitants, dont $55 \%$ de Bouriates et $41 \%$ de Russes), il correspond sensiblement à l'ancienne zone de nomadisation des éleveurs.

Le nomadisme et la vie sous la yourte ont perduré jusque dans les années 1960, dans le contexte de l'économie socialiste. Les premières entreprises de collectivisation de l'élevage eurent lieu au début des années

33. Le statut de «sujet » de la Fédération de Russie est possédé par des entités territoriales aussi différentes que les républiques, des districts (okrug) ou des villes (Moscou et Saint-Petersbourg). Officiellement égaux en droit, les sujets ont leur propre législation, et disposent chacun de deux représentants au Conseil de la Fédération. Les Bouriates sont "titulaires» (i.e. constituent la nationalité éponyme) de trois sujets : une république et deux districts autonomes, dont celui d'Aga. 
1930, les premiers kolkhozes furent créés en 1936-1937. Les informations précises et fiables manquent sur cette période marquée par les répressions staliniennnes, mais il apparaît certain que la transition au socialisme ne fut pas un fleuve tranquille. En revanche, la sédentarisation s'est opérée de manière progressive et en quelque sorte naturelle. Les stajanka d'hiver et d'été, bergeries « en dur " (c'est-à-dire en bois) dispersées sur la steppe, ont petit à petit remplacé les yourtes, tandis qu'autour des bâtiments publics les villages grossissaient des maisons destinées aux kolkhoziens.

Si la deuxième transition, celle de l'abandon du socialisme, fut moins violente que la première, elle n'en a pas moins pris des formes extrêmement brutales et s'apparente à bien des égards à une véritable débâcle organisationnelle et économique. Les statistiques, en l'occurrence, sont éloquentes. Sur ce territoire dont l'élevage a de tout temps constitué la mono-activité, le nombre d'ovins est passé de 771500 à 189000 entre 1991 et 2000. (Le déclin est moindre pour les bovins, passés de 98400 à 70900 têtes.) Cependant, contre vents et marées, la majorité des exploitations collectives s'est maintenue, ou a été maintenue, à coups de subventions. Certes, la place du kolkhoze dans la vie sociale et économique locale s'est radicalement modifiée. Le temps n'est plus où il se confondait avec l'entité villageoise, où le village même procédait de lui. Mais son rôle dans la transition n'en est pas moins essentiel. Tout d'abord, il constitue une sorte de filet de sécurité face aux aléas de la période. En maintenant un certain emploi et une certaine production, il assure aux villageois, ou au moins à une grande partie d'entre eux, un niveau minimal de subsistance. Par ailleurs, il constitue, par ses aides directes ou indirectes, un point d'appui pour le développement d'une activité privée et contribue à la formation d'une manière d'économie mixte. À côté du troupeau kolkhozien gardé par les bergers sur les stajanka existe ainsi un troupeau privé, dont une partie appartient aux bergers eux-mêmes.

Si la plupart des fermes privées agropastorales créées, après 1990, par d'anciens kolkhoziens à la faveur des mesures de privatisation ont des difficultés à subsister, si beaucoup, même, ont rapidement périclité, quelquesunes ont constitué le socle sur lequel s'est bâtie la réussite d'une sorte $\mathrm{d}^{\prime}$ " entrepreneurs villageois ». Ceux-ci continuent à posséder troupeaux et terres, dont ils s'occupent directement ou, plus souvent, indirectement en donnant leur bétail en garde à des bergers du kolkhoze et en installant des employés ${ }^{34}$ sur leur exploitation. À côté de ce qui demeure le fondement de leur économie domestique, ils pratiquent une activité secondaire qui,

34. La large démonétarisation des rémunérations fait de ces employés plus des métayers que des salariés. 
précisément du fait de sa non-nécessité, permet une accumulation de capital. Il s'agit en l'occurrence d'une activité opportuniste liée au transport. Ayant acquis des camions au moment de la privatisation, en empruntant dans des conditions favorables (c'est-à-dire à des taux modérés, à la veille d'une période de forte inflation), ils commercent depuis lors avec la Chine, dont la frontière se trouve seulement à quelque deux cents kilomètres. Ils ont dans un premier temps livré à la ville frontalière de Manjouli des chargements de ferraille provenant du démantèlement partiel du secteur collectif. Ce trafic ayant fini par être interdit, ils exploitent et vont vendre à Manjouli le bois brûlé dans les incendies récurrents de la forêt sibérienne. Chacun est conscient de la précarité foncière de la ressource, à laquelle une période de moindre sécheresse ou une mesure d'interdiction semblable à celle qui a concerné la ferraille peuvent mettre un terme. L'hypothèse d'une future voie reliant Vladivostok à l'Asie centrale laisse déjà envisager le relais d'autres débouchés pour l'activité routière.

Un certain nombre d'éléments permettent de parler d'une « domination locale» des anciens nomades bouriates dans le territoire d'Aga. Celle-ci est d'abord économique. Les entrepreneurs villageois évoqués ci-dessus emploient une main-d'œuvre bouriate (notamment familiale), mais aussi russe (en particulier pour les tâches les plus ingrates, comme l'abattage du bois brûlé), alors que l'inverse n'est pas vrai : il n'y a pas d'entrepreneur russe dans les villages. Elle est également politique. Le pouvoir local (villageois) et régional (au niveau de l'okrug) est aux mains des Bouriates. Il est utilisé pour aider de façon concrète les éleveurs à vivre, voire à maîtriser, la transition. Le maintien de la majorité des kolkhozes, contre vents et marées réformistes, est le fruit d'une volonté politique explicitement orientée vers la protection d'une population à l' habitus collectiviste. ("Ici, on ne peut survivre seul", nous affirmera le ministre de l'agriculture de l'okrug ${ }^{35}$.) Les responsables locaux utilisent les moyens collectifs pratiquement à discrétion pour aider les villageois dans leur vie quotidienne, arrangements qui peuvent également bénéficier aux initiatives des entrepreneurs individuels. Ce soutien informel, ajouté à l'appui régulier que les fermiers trouvent dans le kolkhoze (location de matériels et de terres, gardiennage du bétail sur les stajanka kolkhoziennes), fait apparaître le maintien (politique) des structures collectives comme un facteur déterminant de l'apparition et du développement d'une nouvelle élite économique bouriate ${ }^{36}$.

35. Propos recueillis en septembre 2003.

36. La situation catastrophique d'un des rares villages de l'okrug où le kolkhoze a disparu le montre a contrario. Cf. J.-F. Gossiaux, «Le troupeau et les camions. Processus de transition chez les éleveurs d'Aga ", à paraître dans un ouvrage collectif dirigé par A. Bourgeot, Éd. de la Maison des sciences de l'homme. 
Les dirigeants d'Aga assoient idéologiquement leur pouvoir sur une stratégie ethniciste. Celle-ci se fonde elle-même sur la religion, en l'occurrence le bouddhisme, qui distingue spécifiquement les Bouriates du reste de la population et auquel lesdits dirigeants manifestent une allégeance spectaculaire. (Le portrait du Dalaï Lama côtoie ainsi celui de Vladimir Poutine dans le bureau du gouverneur.) L'identité bouriate est promue et mise en scène. Les représentations emblématiques des clans traditionnels, tirés de l'oubli, s'alignent face au palais du gouvernement régional. Le territoire a tissé des liens institutionnels avec des associations bouriates de Mongolie et de Chine, et il participe activement au festival pan-bouriate (Altargana) organisé tous les deux ans. Il a notamment accueilli celui de 2002.

Les leaders de l'okrug ont exploité au maximum la politique eltsinienne de décentralisation ethnique. Cependant, la nouvelle ligne instaurée par Vladimir Poutine tend vers une recentralisation et une désethnicisation des pouvoirs régionaux. À court terme, le territoire devrait perdre son autonomie et son statut de sujet de la Fédération de Russie. Mais les Bouriates d'Aga et leurs dirigeants sont dans leur grande majorité résolument poutiniens. Quelque regret que leur inspire cette évolution, ils prendront leur part du nouveau système.

Il y a loin des montagnes balkaniques aux steppes sud-sibériennes. Le postsocialisme n'a pas les mêmes couleurs au voisinage occidental de la Chine et aux marges orientales de l'Europe. Dans les Balkans mêmes, il n'est pas indifférent d'être un ex-Yougoslave ou un quasi-Grec. Tout concourt à faire diverger de plus en plus les vies après le nomadisme. Pourtant, nous avons vu qu'il existe entre Valaques, Saracatsans et Bouriates, au-delà des différences passées et présentes, un ensemble de similarités, au premier rang desquelles cette suprématie locale que nous évoquions en entrée et qui s'éclaire, précisément, à la lumière des autres traits communs. Parmi ceux-ci, et sans lien autre qu'indirect avec la question, on notera la sorte d'antécédent historique constituée par les statuts particuliers et les privilèges que les anciens régimes - de l'empire ottoman comme de l'empire russe - accordaient à certains peuples de pasteurs nomades.

Les intéressés eux-mêmes reconnaissent et revendiquent une supériorité discrète qu'ils tendent à généraliser et à expliquer objectivement, sinon à théoriser. Un élément d'explication est leur « débrouillardise ", dont l'affirmation est associée à celle d'un certain mépris des sédentaires, qui en seraient par nature dépourvus. Cette débrouillardise s'affiche d'abord dans le domaine matériel et technique. Et, effectivement, la compétence polyvalente qui est nécessaire dans toute situation d'autarcie l'est plus encore 
dans les conditions de la transhumance (ou de toute forme de nomadisme). Mais la débrouillardise est également, et surtout, sociale. Le pasteur nomade, dans un environnement sédentaire, se trouve constamment en posture d'échange et de négociation : transactions commerciales, organisation des déplacements, relations avec les autorités et l'administration... Une conséquence (et une condition) en est, entre autres, le développement d'une compétence linguistique multiple, en particulier dans une région aussi panachée que les Balkans. Les chefs de famille peuvent être amenés à pratiquer plusieurs langues vernaculaires, en plus de leur langue propre, et une langue véhiculaire.

Le pastoralisme nomade sécrète un certain type de dispositions, au sens que Bourdieu donne à ce terme, dont la portée symbolique est d'autant plus grande qu'elles sont assignées à un mode de vie devenu lui-même emblématique d'une culture (à défendre) ${ }^{37}$. Ces dispositions "traditionnelles " s'actualisent en qualités efficientes dans les sociétés en transition où ce mode de vie s'achève. La débrouillardise matérielle et sociale prend les traits de l'opportunisme économique : elle devient la capacité (et la volonté) de faire feu de tout bois, d'occuper tous les créneaux d'activité dès que ceux-ci apparaissent, fût-ce provisoirement. Ainsi peut s'amorcer un processus d'accumulation (primitive) du capital - à distinguer radicalement du capitalisme de captation dans lequel s'est reconvertie, pour partie, l'ancienne classe dirigeante socialiste. Cet opportunisme peut faire fi de toute retenue statutaire, comme le montre l'exemple des notables karakatchans de Bulgarie allant se faire embaucher comme travailleurs saisonniers en Grèce. La capacité à "aller au charbon » - pour rester dans les métaphores calorifiques - en bousculant les repères de classe reflète le caractère culturellement non pertinent de ceux-ci pour les nomades. La débrouillardise sociale, par ailleurs, ne donne sa pleine efficacité qu'appuyée sur une certaine maîtrise des instruments intellectuels (voire de la " haute culture », pour reprendre l'expression de Gellner) de la société, ou des sociétés, où l'on se meut. La valorisation de l'instruction et de l'excellence intellectuelle procède logiquement de cette nécessité. Dans le contexte postnomade, elle se traduit par un investissement maximal des enfants dans l'institution éducative ${ }^{38}$.

Un certain nombre d'activités économiques couramment associées au pastoralisme nomade représentent autant de ressources transposables dans la société industrielle et capitaliste. Il en est ainsi du commerce au long

37. Cf. "Le modèle valaque», in Gossiaux (2002 : chap. VIII).

38. Cet investissement apparaît efficace chez les Valaques et les Karakatchans. La situation scolaire des jeunes Bouriates semble plus difficile - ce qui valorise d'autant plus les cas de réussite. 
cours, dont nous avons vu l'importance en particulier chez les Valaques aroumains. La maîtrise, inhérente au nomadisme, des pratiques techniques et sociales du transport ${ }^{39}$ peut de même se convertir plus ou moins directement en compétence opérante dans le monde contemporain. Enfin, et indépendamment même de la traditionnelle dispersion systématique des installations commerciales valaques, la multiplicité naturelle des points de sédentarisation est susceptible de constituer l'armature de réseaux nationaux et transnationaux politiquement et économiquement précieux.

Les éléments que nous avons jusqu'ici mis en regard de la supériorité locale des populations considérées, avec une portée potentiellement explicative, ne sont pas liés à la spécificité historique de celles-ci, à savoir l'épisode socialiste qu'elles ont les unes et les autres, de façon certes différenciée, vécu. Cependant, cette spécificité prend, dans la période présente, la forme d'une transition venant s'ajouter à la transition globale qu'est la mondialisation. Et dans la mesure où, comme nous l'avons vu, certains de ces éléments sont surtout efficients, précisément, en situation de transition, on peut la voir comme un facteur explicatif supplémentaire du phénomène. Mais, à l'inverse, le passage au socialisme a dans tous les cas signifié une rupture plus ou moins radicale, et la collectivisation a marqué une perte irrémédiable de statut. On pourrait donc tout aussi bien voir dans l'épisode socialiste un handicap historique.

En fait, nonobstant cette perte de statut, l'organisation collectiviste de l'élevage n'avait pas signifié pour l'ensemble des pasteurs un renversement de leur position sociale. Leur compétence technique les avait naturellement amenés à un certain niveau de responsabilité et de reconnaissance : le berger figurait au rang des travailleurs hautement qualifiés et régulièrement honorés de l'ordre socialiste. Parfois, nous en avons décrit un exemple, la situation après la collectivisation avait gardé les traces de la situation économique antérieure, les possesseurs de bétail devenant les dirigeants d'une coopérative dont ils figuraient comme les initiateurs. Souvent, ils s'étaient retrouvés à occuper individuellement des positions élevées dans l'appareil communiste ${ }^{40}$.

Ce dernier fait renvoie à un trait dont nous avons déjà noté sinon la généralité, du moins la récurrence chez les populations évoquées: le conformisme politique, un légitimisme qui les amène à se situer tout naturellement du côté du pouvoir. "Naturellement » ne signifie cependant pas

39. Nous avons vu que parmi les multiples acceptions du terme vlah figure celle de caravanier.

40. Les éleveurs bouriates, globalement considérés comme koulaks, ont été une cible privilégiée des persécutions staliniennes. À la suite du processus de réhabilitations, les enfants de "réprimés " ont souvent, par une sorte d'effet de compensation, été promus au rang de communistes éminents (héros du travail notamment). 
"tranquillement». Une des modalités de ce légitimisme a ainsi été la loyauté à l'égard des différents États-nations dans lesquels elles ont été intégrées (et entre lesquels elles ont été éventuellement divisées) au hasard de leurs parcours ordinaires; une loyauté qui a pu prendre la forme du nationalisme et de l'activisme, avec les risques afférents. Le légitimisme ressortit à un opportunisme politique qui ressortit lui-même, au même titre que l'opportunisme économique, à la "débrouillardise " nomade, faite de prise de risques et d'anticipation aussi bien que de routine. Ladite débrouillardise apparaît en l'occurrence comme le mode d'encastrement du politique et de l'économique dans le culturel - compris comme mode de vie - et au bout du compte dans l'ethnique ${ }^{41}$.

L'opportunité politique, au tournant des $\mathrm{XX}^{\mathrm{e}}$ et XXI $\mathrm{X}^{\mathrm{e}}$ siècles, a sans doute changé de visage. Quel que soit le sort effectivement dévolu au modèle de l'État-nation, le nouvel ordre mondial, tel qu'il apparaît édicté par les institutions internationales, tend à valoriser le "principe des minorités " à côté, voire à l'encontre, du principe des nationalités sur lequel avait été fondée aux XIX et XX siècles l'organisation politique de l'espace. À cet égard, l'ethnicisme, c'est-à-dire la valorisation et l'utilisation politiques de l'ethnicité, constitue assurément une orientation opportune, dans ses applications infra- aussi bien que transnationales ${ }^{42}$. Les nomades avaient pu conserver des positions favorables dans des formes politico-spatiales - l'État-nation, les Fédérations soviétique et russe - conçues en termes de territorialité et de sédentarité. Une ironie de l'histoire a fait coïncider le déclin sinon la mort annoncée de ces formes a priori peu adaptées aux réalités nomades avec la disparition plus ou moins totale de ces réalités. Le nomadisme comme mode de vie et comme référence culturelle ressortit au passé. Le temps inflige aux "frontières ethniques » des dommages dont l'exploitation des ressources politiques nouvelles représente un mode de réparation, permettant à la singularité efficace du postnomadisme de perdurer dans le monde sédentaire.

École des hautes études en sciences sociales Institut interdisciplinaire d'anthropologie du contemporain, Paris jean-francois.gossiaux@msh-paris.fr

41. L'ethnique lui-même ayant en l'occurrence un fondement intrinsèquement économique et professionnel, comme le montre notamment la polysémie du terme vlah. Sur les rapports de l'ethnique et du culturel et sur la référence au pastoralisme nomade chez les Valaques aroumains, cf. supra, note 37.

42. Cf. «Logique ethnique et ordre politique», in Gossiaux (2002 : conclusion). 
MOTS CLÉS/KEYWORDS: nomadisme/nomadism - transition - Valaques/Vlach Saracatsans/Karakatchan - Bouriates/Bouriat.

RÉFÉRENCES BIBLIOGRAPHIQUES

Abélès, Marc

1992 La Vie quotidienne au Parlement européen. Paris, Hachette.

\section{Antonijevic, Dragoslav}

1982 Obredi i obicaji balkanskih stocara (= Rites et coutumes des pasteurs des Balkans). Belgrade, Balkanoloski Institut.

\section{Bayart, Jean-François}

2004 Le Gouvernement du monde. Une critique politique de la globalisation. Paris, Fayard.

\section{Burawoy, Michael \& Katherine Verdery}

1999 Uncertain Transition. Ethnographies of Change in the Postsocialist World. Lanham, Rowman \& Littlefield.

Cahiers du Centre d'étude des civilisations de l'Europe centrale et du Sud-Est 1989, 8: Les Aroumains. Paris, Publications des Langues' $\mathrm{O}$.

\section{Geertz, Clifford}

1986 Savoir local, savoir global. Les lieux du savoir. Paris, PUF. [Éd. orig. : Local Knowledge. Further Essays in Interpretative Anthropology, New York, Basic Books, 1983.]

\section{Godelier, Maurice}

1990 "La théorie de la transition chez Marx ", Sociologie et sociétés 22 (1) : 53-81.

\section{Gossiaux, Jean-François}

1992 Avoir seize ans dans les Ardennes.

Paris, Éditions du CTHS.

2002 Pouvoirs ethniques dans les Balkans.

Paris, PUF.

Hamel, Jacques \& Mohammed Sfia

1990 "Sur la transition », Sociologie et sociétés 22 (1) : 5-14.

Humphrey, Caroline

1983 Karl Marx Collective : Economy,

Society and Religion in a Siberian Collective Farm. Cambridge, Cambridge University Press /Paris, Maison des sciences de l'homme.

\section{Kavadias, Georges B.}

1965 Pasteurs-nomades méditerranéens.

Les Saracatsans de Grèce. Paris, GauthierVillars.

\section{Polanyi, Karl \& Conrad Arensberg, eds}

1975 Les Systèmes économiques dans l'histoire et dans la théorie. Paris, Larousse. [Éd. orig. : Trade and Market in the Early Empires. Economies in History and Theory, Glencoe, Free Press, 1957.] 
Jean-François Gossiaux, “Il n'existe pas de pauvres chez nous". De la supériorité des nomades sur les sédentaires. - Selon quelle logique des populations nomades, a priori infériorisées par des représentations et des institutions politiques fondées sur la sédentarité, se retrouvent-elles dans une situation de suprématie locale lorsqu'elles sont passées à l'état d'ex-nomades ? À partir de trois exemples, les Valaques et les Karakatchans dans les Balkans, et les Bouriates d'Aga en Sibérie méridionale, l'article analyse les modalités d'une telle suprématie et montre comment le pastoralisme nomade sécrète un certain nombre de dispositions - techniques, économiques, politiques - globalement subsumables sous la notion d'opportunisme, qui peuvent s'actualiser en qualités efficientes dans les sociétés en transition où ce mode de vie s'achève.
Jean-François Gossiaux, "There are no poor among us". On the Superiority of Nomads over the Sedentary. - According to what "logic" do nomadic peoples, who are normally seen as inferior by political institutions grounded on a settled way of life, find themselves in a situation of local supremacy when they become "former nomads " ? Three examples - the Vlach and Karakatchan in the Balkans and the Aga Buriat in southern Siberia serve to analyze this supremacy and show how nomadic pastoralism secretes technical, economic and political conditions that, subsumed under the label of opportunism, can be activated as operational qualities in societies undergoing a transition from nomadism. 